\title{
microRNA-218 expression and its association with the clinicopathological characteristics of patients with cervical cancer
}

\author{
BEI-BEI TANG ${ }^{1}$, SHUI-YI LIU ${ }^{1}$, YU ZHAN ${ }^{1}$, LI-QING WEI ${ }^{1}$, XIAO-LU MAO ${ }^{1}$, \\ JIN WANG ${ }^{1}, \mathrm{LI} \mathrm{LI}^{2}$ and ZHONG-XIN LU ${ }^{1}$ \\ Departments of ${ }^{1}$ Clinical Laboratory and ${ }^{2}$ Gynecology and Obstetrics, The Central Hospital of Wuhan, \\ Tongji Medical College, Huazhong University of Science and Technology, Wuhan, Hubei 430014, P.R. China
}

Received July 30, 2014; Accepted April 13, 2015

DOI: $10.3892 /$ etm.2015.2455

\begin{abstract}
The aim of the present study was to investigate the expression of microRNA-218 (miRNA-218) in the serum and cervical tissue and its association with the clinicopathological features of cervical cancer (CC). The expression of miRNA-218 was detected in the serum and cervical tissue of 112 patients with $\mathrm{CC}$ and 50 age-matched hysteromyoma patients via the reverse transcription-quantitative polymerase chain reaction. The clinical data were collected and the association between the expression of miRNA-218 and the clinicopathological characteristics of the patients was analyzed. The expression of miRNA-218 in the cancer group was significantly decreased in the cervical tissue and serum compared with that in the control group $(\mathrm{P}<0.001)$. The decreased expression of miRNA-218 was associated with a later International Federation of Gynecology and Obstetrics stage, a more invasive pathological type and lymphatic node metastasis but not with age, age at menarche, menopausal status, number of pregnancies and deliveries, family history of cancer or tumor size. In conclusion, miRNA-218 was found to be downregulated in the cancer tissue and serum of the patients with CC. The decreased expression of miRNA-218 in CC was associated with the invasiveness of the tumor.
\end{abstract}

\section{Introduction}

microRNAs (miRNAs) are a group of single-stranded RNAs composed of $\sim 22$ nucleotides that control numerous developmental and cellular processes in eukaryotic organisms $(1,2)$.

Correspondence to: Dr Bei-Bei Tang, Department of Clinical Laboratory, The Central Hospital of Wuhan, Tongji Medical College, Huazhong University of Science and Technology, 26 Shengli Street, Wuhan, Hubei 430014, P.R. China

E-mail: tangbeibei2104@163.com

Key words: cervical cancer, microRNA-218, cancer tissue, serum, clinicopathological characteristics
Previous studies have identified major factors participating in the biogenesis of miRNAs and have established the basic principles of their function. At present, miRNAs are considered to be master regulators of a number of important biological processes, including cell growth and apoptosis, viral infections and cancer development (3-6). Furthermore, several studies have reported that miRNAs are promising biomarkers for the classification and outcome prediction of several types of human cancer, such as lung (7), breast (8), colon (9) and cervical (10) cancer.

Cervical cancer (CC) is the second most common cause of cancer-related mortality in women worldwide, resulting in $\sim 300,000$ deaths annually $(10,11)$. Despite the fact that most patients with $\mathrm{CC}$ receive standard treatments, including radiotherapy, chemotherapy and surgical intervention, the clinical outcomes of CC vary markedly and are difficult to predict. A previous study found that CC tissues had altered miRNA expression compared with adjacent normal tissues (12). In addition, the prognostic value of several miRNAs in CC has already been determined $(10,13)$. Yu et al (14) reported that the circulating miRNA-218 level was reduced in CC and correlated with tumor invasion; however, to date, the expression of miRNA-218 in CC tissue and its prognostic value have not yet been investigated. The present study aimed to examine the expression of miRNA-218 in the serum and cancer tissue of patients with CC and to investigate its possible correlation with certain clinicopathological features, such as tumor size, stage and metastasis.

\section{Materials and methods}

Patients and samples. A total of 112 patients pathologically diagnosed with CC, who had been admitted to The Central Hospital of Wuhan (Wuhan, China) between June 2010 and June 2011, were enrolled in this study. None of the patients received either radiotherapy or chemotherapy prior to surgery. Fifty age-matched hysteromyoma patients were selected as the control group. Cancer tissue samples from the patients with $\mathrm{CC}$, normal cervix tissue samples from the patients with hysteromyoma and serum samples from the two groups were collected for miRNA-218 detection. The blood samples were collected 
Table I. Clinicopathological features of the cancer and control groups.

\begin{tabular}{|c|c|c|c|}
\hline Variables & Cancer group, $n=112$ & Control group, $n=50$ & P-value \\
\hline Age $\left(\right.$ years) ${ }^{\mathrm{a}}$ & $42.4 \pm 7.5$ & $40.0 \pm 10.2$ & 0.15 \\
\hline Age at menarche (years) ${ }^{\mathrm{a}}$ & $15.5 \pm 3.2$ & $14.8 \pm 2.5$ & 0.20 \\
\hline Menopausal status & & & 0.85 \\
\hline Yes & 32 & 15 & \\
\hline No & 80 & 35 & \\
\hline Pregnancies (n) & & & 0.85 \\
\hline $0-1$ & 82 & 38 & \\
\hline$\geq 2$ & 30 & 12 & \\
\hline Deliveries (n) & & & 0.52 \\
\hline $0-1$ & 88 & 42 & \\
\hline$\geq 2$ & 24 & 8 & \\
\hline Family history of cancer & & & 0.50 \\
\hline Yes & 6 & 4 & \\
\hline No & 106 & 46 & \\
\hline FIGO stage & & & N/A \\
\hline $\mathrm{I} / \mathrm{II}$ & 75 & N/A & \\
\hline III/IV & 37 & N/A & \\
\hline Tumor size (cm) & & & $\mathrm{N} / \mathrm{A}$ \\
\hline$<4$ & 68 & $\mathrm{~N} / \mathrm{A}$ & \\
\hline$\geq 4$ & 44 & N/A & \\
\hline Lymph node metastasis & & & N/A \\
\hline Yes & 38 & $\mathrm{~N} / \mathrm{A}$ & \\
\hline No & 74 & $\mathrm{~N} / \mathrm{A}$ & \\
\hline
\end{tabular}

${ }^{\mathrm{a} D a t a}$ are expressed as the mean \pm standard deviation. N/A, not applicable; FIGO, International Federation of Gynecology and Obstetrics.

prior to any treatment and were centrifuged $(10,000 \mathrm{x} \mathrm{g}$ for 5 min at $4^{\circ} \mathrm{C}$ ) immediately. Tissue samples, including $\mathrm{CC}$ and normal cervix tissues were collected directly in the operating room. All tissue samples were pathologically confirmed under light microscope. The blood and tissue samples were stored at $-80^{\circ} \mathrm{C}$ until they were analyzed. The clinicopathological features, such as age, histological subtype, International Federation of Gynecology and Obstetrics (FIGO) stage, tumor size, lymph node metastasis, age at menarche, menopausal status, number of pregnancies and deliveries and family history of cancer, of the patient group and, if appropriate, the control group were evaluated. The present study was approved by the Ethics Committee of The Central Hospital of Wuhan and all patients provided written informed consent.

miRNA-218 analysis using the reverse transcription-quantitative polymerase chain reaction ( $R T-q P C R)$. The details of the sample preparation are described in the 'Patients and samples' section. Total RNA was isolated using TRIzol ${ }^{\circledR}$ Reagent (Invitrogen Life Technologies, Carlsbad, CA, USA). The mirVana $^{\mathrm{TM}}$ miRNA Isolation kit (Applied Biosystems, Foster City, CA, USA) was used for the isolation of the serum miRNA, as previously described (14). The expression of miRNA-218 was determined using an miRNA -218 Taqman miRNA assay kit (Invitrogen Life Technologies), according to the manufacturer's instructions. U6 RNA (Ruibo Biotechnology Corp., Guangzhou, China) was used as an endogenous control for the data analysis. The difference in the expression of miRNA-218 between the CC group and the control group was calculated with the $2^{-\Delta \Delta C t}$ method, as previously described (15). All procedures were repeated three times.

Statistical analysis. Data are expressed as the mean \pm standard deviation or as numbers and percentages. The association between the miRNA-218 expression and the clinicopathological features of CC was tested using one-way analysis of variance. The Mann-Whitney U test was used to determine the difference in the miRNA-218 levels between the two groups. Statistical analysis was performed using SPSS software, version 18.0 (SPSS Inc., Chicago, IL, USA). A two-tailed $\mathrm{P}<0.05$ was considered to indicate a statistically significant difference.

\section{Results}

Clinicopathological characteristics. The clinicopathological characteristics of the 112 patients with CC and the 50 age-matched patients with hysteromyoma are shown in Table I. The mean age of the patients with CC and hysteromyoma was 42.4 and 40.0 years, respectively. On the basis of 
A

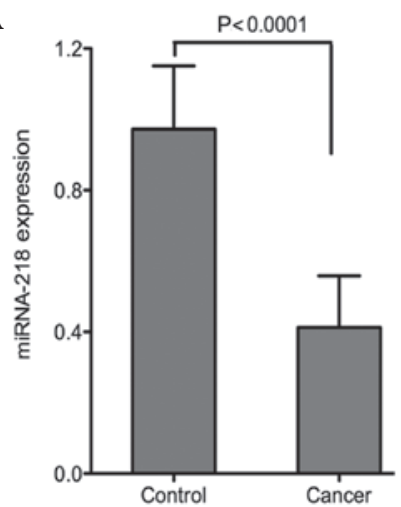

B

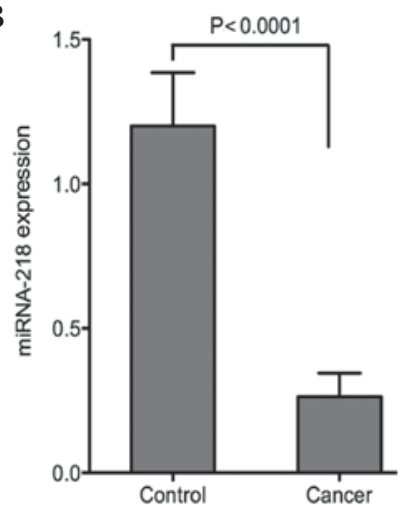

Figure 1. Expression of miRNA-218 in the (A) serum and (B) cervical tissue of patients with cervical cancer. The miRNA-218 expression in the serum and cervical tissue of the cancer group was significantly decreased compared with that of the control group. miRNA-218, microRNA-218.

A

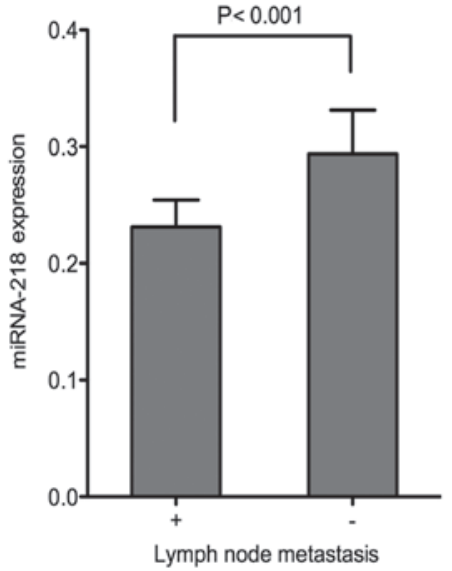

C

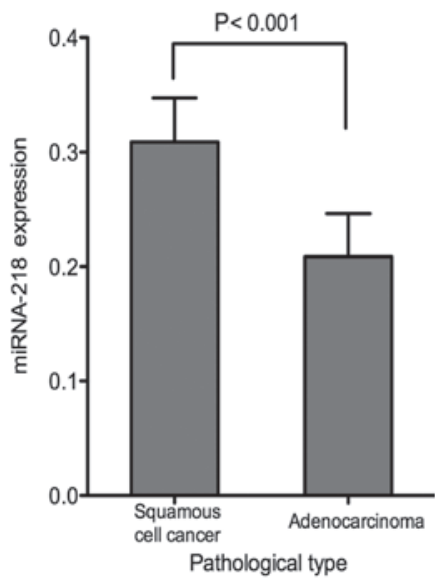

B

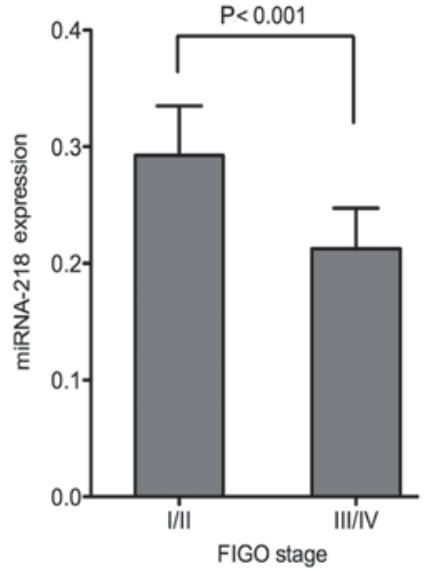

D

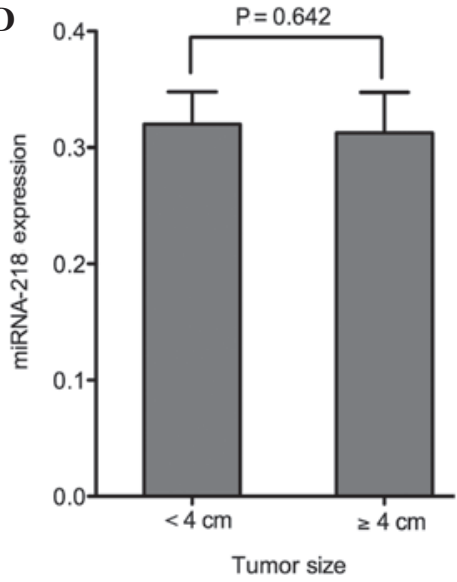

Figure 2. Association between miRNA-218 expression in CC tissue and (A) lymph node metastasis, (B) tumor stage, (C) pathological type and (D) tumor size. Patients with lymph node metastasis, later FIGO stages and more invasive pathological type exhibited lower expression of miRNA-218 in the CC tissue. The miRNA-218 expression in cancer tissue was not associated with tumor size. miRNA-218, microRNA-218; FIGO, International Federation of Gynecology and Obstetrics; CC, cervical cancer.

archived pathology reports, there were 98 cases of squamous cell cancer (98/112) and 14 cases of adenocarcinoma (14/112).

Among the 112 patients with CC, 75 exhibited FIGO stage I/II (75/112) and 37 had stage III/IV (37/112) tumors. Lymph node metastasis was detected in 38/112 cases. No significant differences were found in age, age at menarche, menopausal status, number of pregnancies and deliveries or family history of cancer between the $\mathrm{CC}$ and control groups $(\mathrm{P}>0.05)$.
Expression of miRNA-218 in the serum and tissue in the cancer and normal samples. The expression of miRNA-218 in the serum and tissue was analyzed using RT-qPCR. Compared with the control group, the expression of miRNA-218 in the serum was significantly decreased in the patients with CC $(\mathrm{P}<0.0001)$. The expression of miRNA-218 in the normal cervical tissue was significantly higher than that in the cancer tissue ( $\mathrm{P}<0.0001$; Fig. 1). 
Table II. miRNA-218 expression in different subgroups of patients with cervical cancer $(\mathrm{n}=112)$.

\begin{tabular}{|c|c|c|c|c|}
\hline Variables & Relative expression (Serum) & P-value & Relative expression (Tissue) & P-value \\
\hline Age, years ${ }^{\mathrm{a}}$ & & 0.24 & & 0.35 \\
\hline$\leq 30(\mathrm{n}=12)$ & $0.39 \pm 0.12$ & & $0.26 \pm 0.08$ & \\
\hline $30-45(n=55)$ & $0.42 \pm 0.10$ & & $0.30 \pm 0.10$ & \\
\hline$\geq 45(n=45)$ & $0.40 \pm 0.10$ & & $0.28 \pm 0.11$ & \\
\hline Age at menarche, years ${ }^{\mathrm{a}}$ & & 0.10 & & 0.23 \\
\hline$\leq 12(\mathrm{n}=20)$ & $0.40 \pm 0.05$ & & $0.28 \pm 0.10$ & \\
\hline $12-14(n=68)$ & $0.38 \pm 0.10$ & & $0.27 \pm 0.06$ & \\
\hline$\geq 14(n=24)$ & $0.41 \pm 0.13$ & & $0.30 \pm 0.08$ & \\
\hline Menopausal status & & 0.18 & & 0.10 \\
\hline Yes $(n=32)$ & $0.42 \pm 0.12$ & & $0.28 \pm 0.06$ & \\
\hline No $(n=80)$ & $0.40 \pm 0.08$ & & $0.31 \pm 0.10$ & \\
\hline Pregnancies, $n$ & & 0.22 & & 0.15 \\
\hline $0-1(n=82)$ & $0.39 \pm 0.09$ & & $0.29 \pm 0.10$ & \\
\hline$\geq 2(\mathrm{n}=30)$ & $0.41 \pm 0.10$ & & $0.30 \pm 0.08$ & \\
\hline Deliveries, $\mathrm{n}$ & & 0.11 & & \\
\hline $0-1(n=88)$ & $0.42 \pm 0.14$ & & $0.32 \pm 0.07$ & 0.20 \\
\hline$\geq 2(n=24)$ & $0.39 \pm 0.08$ & & $0.29 \pm 0.08$ & \\
\hline Family history of cancer, $\mathrm{n}$ & & 0.15 & & 0.16 \\
\hline $0-1(n=6)$ & $0.38 \pm 0.12$ & & $0.28 \pm 0.05$ & \\
\hline$\geq 2(\mathrm{n}=106)$ & $0.41 \pm 0.10$ & & $0.32 \pm 0.09$ & \\
\hline
\end{tabular}

Data are expressed as the mean \pm standard deviation.
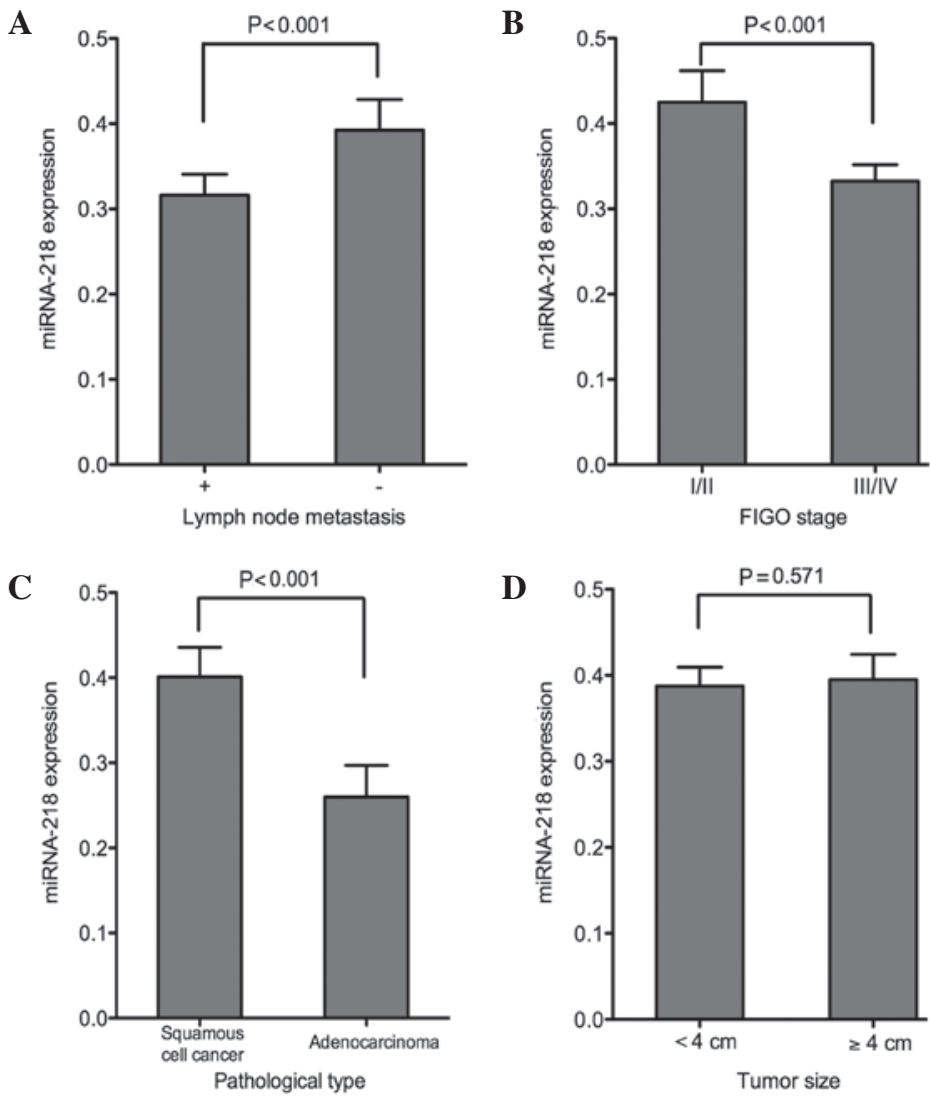

Figure 3. Association between serum miRNA-218 expression and (A) lymph node metastasis, (B) tumor stage, (C) pathological type and (D) tumor size. Patients with lymph node metastasis, later stages and more invasive pathological type exhibited lower expression of miRNA-218 in the serum samples. The expression of miRNA-218 in the serum was not associated with the tumor size. miRNA-218, microRNA-218; FIGO, International Federation of Gynecology and Obstetrics. 
Association between miRNA expression and clinicopathological characteristics. The association between the miRNA-218 expression in the serum and cervical tissue samples and the clinicopathological characteristics of the patients was investigated in the present study. No association was found between miRNA-218 expression and age in the tissue or in the serum samples $(\mathrm{P}>0.05)$. The subgroup analysis on the basis of age at menarche showed similar miRNA-218 expression in both the cancer tissue and the serum $(\mathrm{P}>0.05)$. No significant differences in the miRNA-218 expression were found in subgroup analyses based on menopausal status, number of pregnancies and deliveries and family history of cancer $(\mathrm{P}>0.05)$ (Table II). The expression level of miRNA-218 in the patients with lymph node metastasis was significantly lower than that in the patients without lymph node metastasis $(\mathrm{P}<0.001)$. Significant differences in the serum and tissue miRNA-218 expression levels were found between the earlier- and later-stage subgroups (FIGO stage I/II and III/IV, respectively) $(\mathrm{P}<0.001)$. The patients with squamous cell cancer showed a higher level of miRNA-218 in both the serum and the CC tissue compared with the adenocarcinoma patients $(\mathrm{P}<0.001)$. No association was detected between miRNA-218 levels and tumor size ( $\mathrm{P}>0.05)$ (Figs. 2 and 3).

\section{Discussion}

In the present study, a significant decrease was observed in the miRNA-218 expression levels in the cancer tissue and serum of patients with CC. The decreased expression of miRNA-218 was associated with the pathological type of cancer, tumor stage and lymph node metastasis, which suggested that miRNA-218 may act as a tumor suppressive miRNA in CC.

miRNAs have been reported to suppress or promote tumor metastasis, providing a new perspective on the metastatic process (16). Several miRNAs have been associated with the outcome prediction of CC (10). The present findings showed that the expression of miRNA-218 in the serum was significantly decreased in patients with $\mathrm{CC}$, particularly in patients with a later FIGO stage and lymph node metastasis. These results were consistent with those of previous studies $(9,17)$. Despite the significant decrease in the miRNA-218 expression found in the serum of patients with $\mathrm{CC}$, the change in the serum miRNA-218 levels appeared to be less than that in a previous study (14). This may have been due to the different controls used in each study. In the present study, hysteromyoma patients were used as the control, while age-matched healthy women were used as the control in the previous study (14). Despite the lack of studies reporting the expression of miRNA-218 in hysteromyoma patients, it is possible that the serum expression of miRNA-218 is also decreased in those patients; however, further studies are required for this hypothesis to be confirmed. The present study is the first, to the best of our knowledge, to demonstrate that the expression of miRNA-218 in CC tissue is notably decreased.

It is currently believed that the invasion and metastasis of cancer are the main reasons for high rates of tumor-related mortality. CC patients with regional or distant metastasis have a generally poor prognosis (18). A previous study suggested that the deregulation of miRNAs could play an important role in the malignant transformation of CC (19). In addition, it has been reported that miRNA-218 is deregulated in several types of cancer and that its downregulation is associated with a poor prognosis (20). These findings indicate that miRNA-218 acts as a tumor suppressor.

In the present study, the decreased miRNA-218 expression in the serum and cancer tissue was associated with tumor metastasis and a later FIGO stage. Although numerous studies have attempted to identify pathways through which miRNA-218 can regulate tumor invasion $(21,22)$, the exact mechanism of the decreased expression of miRNA-218 in CC remains unclear.

In conclusion, the serum and tissue levels of miRNA-218 are significantly decreased in patients with $\mathrm{CC}$, and these decreases are significantly associated with the invasiveness of the tumor, a later tumor stage and lymph node metastasis. There is a likelihood that miRNA-218 is a regulator of tumor invasion and metastasis in $\mathrm{CC}$; however, additional studies are required to further elucidate this association and the exact mechanism.

\section{Acknowledgements}

This study was financially supported by the Science and Technology Bureau of Wuhan.

\section{References}

1. Bartel DP: MicroRNAs: Genomics, biogenesis, mechanism and function. Cell 116: 281-297, 2004.

2. Krol J, Loedige I and Filipowicz W: The widespread regulation of microRNA biogenesis, function and decay. Nat Rev Genet 11: 597-610, 2010.

3. Ambros V: The functions of animal microRNAs. Nature 431: 350-355, 2004.

4. Calin GA and Croce CM: MicroRNA signatures in human cancers. Nat Rev Cancer 6: 857-866, 2006.

5. Johnson CD, Esquela-Kerscher A, Stefani G, et al: The let-7 microRNA represses cell proliferation pathways in human cells. Cancer Res 67: 7713-7722, 2007.

6. Miska EA: How microRNAs control cell division, differentiation and death. Curr Opin Genet Dev 15: 563-568, 2005.

7. Yu SL, Chen HY, Chang GC, et al: MicroRNA signature predicts survival and relapse in lung cancer. Cancer Cell 13: 48-57, 2008.

8. Rothé F, Ignatiadis M, Chaboteaux C, et al: Global microRNA expression profiling identifies MiR-210 associated with tumor proliferation, invasion and poor clinical outcome in breast cancer. PLoS One 6: e20980, 2011

9. Cheng H, Zhang L, Cogdell DE, et al: Circulating plasma MiR-141 is a novel biomarker for metastatic colon cancer and predicts poor prognosis. PLoS One 6: e17745, 2011.

10. Hu X, Schwarz JK, Lewis JS Jr et al: A microRNA expression signature for cervical cancer prognosis. Cancer Res 70: 1441-1448, 2010.

11. Kang HW, Wang F, Wei Q, et al: miR-20a promotes migration and invasion by regulating TNKS2 in human cervical cancer cells. FEBS Lett 586: 897-904, 2012.

12. Rao Q, Shen Q, Zhou H, Peng Y, Li J and Lin Z: Aberrant microRNA expression in human cervical carcinomas. Med Oncol 29: 1242-1248, 2012.

13. Huang L, Lin JX, Yu YH, Zhang MY, Wang HY and Zheng M: Downregulation of six microRNAs is associated with advanced stage, lymph node metastasis and poor prognosis in small cell carcinoma of the cervix. PLoS One 7: e33762, 2012.

14. Yu J, Wang Y, Dong R, Huang X, Ding S and Qiu H: Circulating microRNA-218 was reduced in cervical cancer and correlated with tumor invasion. J Cancer Res Clin Oncol 138: 671-674, 2012.

15. Zhang Z, Li Z, Gao C, et al: miR-21 plays a pivotal role in gastric cancer pathogenesis and progression. Lab Invest 88: 1358-1366, 2008.

16. Tie J, Pan Y, Zhao L, et al: MiR-218 inhibits invasion and metastasis of gastric cancer by targeting the Robol receptor. PLoS Genet 6: e1000879, 2010. 
17. Tatarano S, Chiyomaru T, Kawakami K, et al: miR-218 on the genomic loss region of chromosome 4 p15.31 functions as a tumor suppressor in bladder cancer. Int J Oncol 39: 13-21, 2011.

18. Burki TK: Cervical cancer: Screening and risk with age. Lancet Oncol 15: e107, 2014.

19. Lee JW, Choi CH, Choi JJ, et al: Altered microRNA expression in cervical carcinomas. Clin Cancer Res 14: 2535-2542, 2008.

20. Jin J, Cai L, Liu ZM and Zhou XS: miRNA-218 inhibits osteosarcoma cell migration and invasion by down-regulating of TIAM1, MMP2 and MMP9. Asian Pac J Cancer Prev 14: 3681-3684, 2013
21. Uesugi A, Kozaki K, Tsuruta T, et al: The tumor suppressive microRNA miR-218 targets the mTOR component Rictor and inhibits AKT phosphorylation in oral cancer. Cancer Res 71: 5765-5778, 2011.

22. Song L, Huang Q, Chen $\mathrm{K}$, et al: miR-218 inhibits the invasive ability of glioma cells by direct downregulation of IKK- $\beta$. Biochem Biophys Res Commun 402: 135-140, 2010. 